\title{
WEIGHTED HARDY TYPE INTEGRAL INEQUALITIES INVOLVING MANY FUNCTIONS
}

\author{
SABIR HUSSAIN, MUHAMMAD AMER LATIF AND WASEEM AKHTAR
}

\begin{abstract}
The object of this paper is to derive some new weighted Hardy type integral inequalities involving many functions and to obtain a classical weighted Hardy type inequality involving many functions.
\end{abstract}

\section{Introduction}

In the theory of inequalities one of the well known inequality, due to G. H. Hardy, called classical Hardy inequality, is the following [6, Theorem 330]:

If $p>1, f(x) \geq 0$ for $0<x<\infty$ and $R(x)=\frac{1}{x} \int_{0}^{x} f(t) d t$, then

$$
\int_{0}^{\infty} R^{p}(x) d x<\left[\frac{p}{p-1}\right]^{p} \int_{0}^{\infty} f^{p}(x) d x,
$$

unless $f \equiv 0$. The inequality (1) is sharp one, that is, involved constant is the best possible one. It was Hardy who first generalized his own result (1) as:

For $m \neq 1, p>1$ and $f:(0, \infty) \rightarrow(0, \infty)$, integrable function, the following does hold:

$$
\begin{aligned}
& \int_{0}^{\infty} F^{p}(x) d x< {\left[\frac{p}{|m-1|}\right]^{p} \int_{0}^{\infty} x^{p-m} f^{p}(x) d x, } \\
& F(x)= \begin{cases}\int_{0}^{x} f(t) d t & m>1 ; \\
\int_{0}^{\infty} f(t) d t & m<1,\end{cases}
\end{aligned}
$$

unless $f \equiv 0$. Inequality (2) is also sharp. The inequalities (1) and (2) have great importance in the theory and applications of integral inequalities, and in particular in the analysis of qualitative as well as quantitative properties of solutions of differential and integral equations. Due to this, over the years much efforts and time has been devoted to the improvement and generalization of Hardy's inequalities (1) and (2). These includes, among others, the works in $[1,6,7,8,10,11,12,13,14]$ and by Hussain, Pečarić in $[2,3,4,5]$.

Corresponding author: Sabir Hussain.

2010 Mathematics Subject Classification. 26D10, $26 \mathrm{D} 15$.

Key words and phrases. Hardy inequality, Hölder's inequality. 
The main aim of this paper is to establish new weighted Hardy type integral inequalities involving two or more functions and to derive some weighted classical Hardy type inequalities by means of fairly elementary analysis.

\section{Main results}

Lemma 1 ([9]). If $C_{1}, C_{2}, \ldots, C_{n}$ are reals and $C_{n+1}=C_{1}$, then

$$
\sum_{r=1}^{n-k+2} C_{r} C_{r+1} \cdots C_{r+k-1} \leq \sum_{r=1}^{n} C_{r}^{k}, \text { where } n \geq k-1 .
$$

Theorem 1. For any $1 \leq i \leq n$, let $f_{i}(x)$ be a non-negative and integrable function on $(0, X)$, $X \in \mathbb{R}_{+}$and $w, u_{i}, z_{i}:[0, x] \rightarrow \mathbb{R}_{+}$, absolutely continuous with $z_{i}^{\prime}$ essentially bounded and positive (a.e). If $u_{i}$ is increasing and

$$
\begin{aligned}
& 1+\frac{u_{i}(x) w^{\prime}(x)}{(1-2 \alpha) w(x) u_{i}^{\prime}(x)} \geq \frac{1}{\gamma_{i}}>0 \quad(\text { a.e }), \text { for } \alpha>\frac{1}{2} \text { and } 1 \leq i \leq n, \\
& 1+\frac{u_{i}(x) w^{\prime}(x)}{(1-2 \alpha) w(x) u_{i}^{\prime}(x)} \geq \frac{1}{\delta_{i}}>0 \quad(\text { a.e }), \text { for } \alpha<\frac{1}{2} \text { and } 1 \leq i \leq n .
\end{aligned}
$$

Then,

$$
\sum_{i=1}^{n} \int_{0}^{\infty} w(x) R_{i}(x) R_{i+1}(x) d x \leq \sum_{i=1}^{n}\left[\frac{2 \lambda_{i}}{|2 \alpha-1|}\right]^{2} \int_{0}^{\infty} w(x) g_{i}(x) d x
$$

where,

$$
\begin{gathered}
g_{i}(x) \mapsto \frac{u_{i}^{4-2 \alpha}(x)\left[z_{i}^{\prime}(x)\right]^{2} f_{i}^{2}(x)}{z_{i}^{2}(x) u_{i}^{\prime}(x)} ; \quad \lambda_{i}=\max _{1 \leq i \leq n}\left(\gamma_{i}, \delta_{i}\right) \text { and } \\
R_{i}(x)=\left\{\begin{array}{l}
\frac{\sqrt{u_{i}^{\prime}(x)}}{u_{i}^{\alpha}(x)} \int_{0}^{x} \frac{u_{i}(t) z_{i}^{\prime}(t)}{z_{i}(t)} f_{i}(t) d t, \quad \alpha>\frac{1}{2} ; \\
\frac{\sqrt{u_{i}^{\prime}(x)}}{u_{i}^{\alpha}(x)} \int_{x}^{\infty} \frac{u_{i}(t) z_{i}^{\prime}(t)}{z_{i}(t)} f_{i}(t) d t, \quad \alpha<\frac{1}{2} .
\end{array}\right.
\end{gathered}
$$

Proof. Let us define for $\alpha>\frac{1}{2}, 0 \leq x \leq X$ and $0<a<b<\infty$ :

$$
R_{i a}(x)=\frac{\sqrt{u_{i}^{\prime}(x)}}{u_{i}^{\alpha}(x)} \int_{a}^{x} \frac{u_{i}(t) z_{i}^{\prime}(t)}{z_{i}(t)} f_{i}(t) d t, 1 \leq i \leq n,
$$

with $R_{i 0}(x)=R_{i}(x)$.

Using the inequality (3) with $k=2$ for $C_{i}=R_{i a}(x)$ :

$$
\sum_{i=1}^{n} R_{i a}(x) R_{(i+1) a}(x) \leq \sum_{i=1}^{n} R_{i a}^{2}(x) .
$$


Multiplying both sides $w(x)$ and integrating from $a$ to $b$

$$
\sum_{i=1}^{n} \int_{a}^{b} w(x) R_{i a}(x) R_{(i+1) a}(x) d x \leq \sum_{i=1}^{n} \int_{a}^{b} w(x) R_{i a}^{2}(x) d x .
$$

Now,

$$
I=\int_{a}^{b} w(x) R_{i a}^{2}(x) d x=\int_{a}^{b} w(x)\left[\frac{\sqrt{u_{i}^{\prime}(x)}}{u_{i}^{\alpha}(x)} \int_{a}^{x} \frac{u_{i}(t) z_{i}^{\prime}(t)}{z_{i}(t)} f_{i}(t) d t\right]^{2} d x .
$$

Integrating by parts we have

$$
\begin{aligned}
I= & \mid \frac{u_{i}^{1-2 \alpha}(x)}{1-2 \alpha}\left[\left.\left.\sqrt{w(x)} \int_{a}^{x} \frac{u_{i}(t) z_{i}^{\prime}(t)}{z_{i}(t)} f_{i}(t) d t\right|^{2}\right|_{a} ^{b}\right. \\
& -\int_{a}^{b} \frac{u_{i}^{1-2 \alpha}(x)}{1-2 \alpha} \frac{d}{d x}\left[\sqrt{w(x)} \int_{a}^{x} \frac{u_{i}(t) z_{i}^{\prime}(t)}{z_{i}(t)} f_{i}(t) d t\right)^{2} d x \\
= & \frac{w(b) u_{i}^{1-2 \alpha}(b)}{1-2 \alpha}\left[\int_{a}^{b} \frac{u_{i}(t) z_{i}^{\prime}(t)}{z_{i}(t)} f_{i}(t) d t\right]^{2}-\frac{1}{1-2 \alpha} \int_{a}^{b} \frac{u_{i}(x) w^{\prime}(x) R_{i a}^{2}(x)}{u_{i}^{\prime}(x)} d x \\
& +\frac{2}{1-2 \alpha} \int_{a}^{b} \frac{u_{i}^{2-\alpha}(x) w(x) z_{i}^{\prime}(x) f_{i}(x) R_{i a}(x)}{z_{i}(x) \sqrt{u_{i}^{\prime}(x)}} d x,
\end{aligned}
$$

i.e.,

$$
\begin{aligned}
& \int_{a}^{b} w(x) R_{i a}^{2}(x)\left[1+\frac{u_{i}(x) w^{\prime}(x)}{(1-2 \alpha) w(x) u_{i}^{\prime}(x)}\right] d x \\
& \quad \leq \frac{2}{2 \alpha-1} \int_{a}^{b} \frac{u_{i}^{2-\alpha}(x) w(x) z_{i}^{\prime}(x) f_{i}(x) R_{i a}(x)}{z_{i}(x) \sqrt{u_{i}^{\prime}(x)}} d x
\end{aligned}
$$

From (4) and (8) we have

$$
\int_{a}^{b} w(x) R_{i a}^{2}(x) d x \leq \frac{2 \gamma_{i}}{2 \alpha-1} \int_{a}^{b} \frac{u_{i}^{2-\alpha}(x) w(x) z_{i}^{\prime}(x) f_{i}(x) R_{i a}(x)}{z_{i}(x) \sqrt{u_{i}^{\prime}(x)}} d x
$$

By Hölder's inequality:

$$
\begin{aligned}
\int_{a}^{b} w(x) R_{i a}^{2}(x) d x \leq & \frac{2 \gamma_{i}}{2 \alpha-1} \sqrt{\int_{a}^{b}\left[\sqrt{w(x)} R_{i a}(x)\right]^{2} d x} \\
& \times \sqrt{\int_{a}^{b}\left[\frac{u_{i}^{2-\alpha}(x) \sqrt{w(x)} z_{i}^{\prime}(x) f_{i}(x)}{z_{i}(x) \sqrt{u_{i}^{\prime}(x)}}\right]^{2} d x}
\end{aligned}
$$


i.e.,

$$
\begin{aligned}
\int_{a}^{b} w(x) R_{i a}^{2}(x) d x & \leq 4\left[\frac{\gamma_{i}}{2 \alpha-1}\right]^{2} \int_{0}^{b} \frac{u_{i}^{4-2 \alpha}(x) w(x)\left[z_{i}^{\prime}(x)\right]^{2} f_{i}^{2}(x)}{z_{i}^{2}(x) u_{i}^{\prime}(x)} d x \\
& \leq 4\left[\frac{\gamma_{i}}{2 \alpha-1}\right]^{2} \int_{0}^{\infty} \frac{u_{i}^{4-2 \alpha}(x) w(x)\left[z_{i}^{\prime}(x)\right]^{2} f_{i}^{2}(x)}{z_{i}^{2}(x) u_{i}^{\prime}(x)} d x .
\end{aligned}
$$

By letting $b \mapsto \infty$ and from inequalities (7) and (9) we have

$$
\sum_{i=1}^{n} \int_{0}^{\infty} w(x) R_{i}(x) R_{i+1}(x) d x \leq \sum_{i=1}^{n}\left[\frac{2 \lambda_{i}}{2 \alpha-1}\right]^{2} \int_{0}^{\infty} w(x) g_{i}(x) d x .
$$

Let us define for $\alpha<\frac{1}{2}$ and $0<a<b<\infty$,

$$
R_{i b}(x)=\frac{\sqrt{u_{i}^{\prime}(x)}}{u_{i}^{\alpha}(x)} \int_{x}^{b} \frac{u_{i}(t) z_{i}^{\prime}(t)}{z_{i}(t)} f_{i}(t) d t, \quad 1 \leq i \leq n,
$$

with $R_{i \infty}(x)=R_{i}(x)$.

Following the same steps as in the proof of inequality (10) we obtain

$$
\sum_{i=1}^{n} \int_{0}^{\infty} w(x) R_{i}(x) R_{i+1}(x) d x \leq \sum_{i=1}^{n}\left[\frac{2 \lambda_{i}}{1-2 \alpha}\right]^{2} \int_{0}^{\infty} w(x) g_{i}(x) d x .
$$

Inequalities (10) and (11) are equivalent to (6).

Corollary 1. For any $1 \leq i \leq n$, let $f_{i}(x)$ be a non-negative and integrable function on $(0, X)$, $X \in \mathbb{R}_{+}, \lambda_{i}=\max _{1 \leq i \leq n}\left(\gamma_{i}, \delta_{i}\right)$ and $w:[0, X] \rightarrow \mathbb{R}_{+}$, absolutely continuous. Let

$$
\begin{aligned}
& 1-\frac{x w^{\prime}(x)}{w(x)} \geq \frac{1}{\gamma_{i}}>0, \text { for } \alpha>\frac{1}{2} \text { and } 1 \leq i \leq n, \\
& 1+\frac{x w^{\prime}(x)}{w(x)} \geq \frac{1}{\delta_{i}}>0, \text { for } \alpha<\frac{1}{2} \text { and } 1 \leq i \leq n .
\end{aligned}
$$

Then,

$$
\sum_{i=1}^{n} \int_{0}^{\infty} w(x)\left[\frac{1}{x} \int_{0}^{x} f_{i}(t) d t\right]^{2} d x \leq 4 \sum_{i=1}^{n} \lambda_{i}^{2} \int_{0}^{\infty} w(x) f_{i}^{2}(x) d x .
$$

Proof. Follows from Theorem 1 by setting $z_{i}(t)=u_{i}(t)=t ; \alpha=1$ and $f_{i}(t)=f_{i+1}(t)$ for $1 \leq i \leq$ $n$.

Theorem 2. Let the conditions of Theorem 1 be satisfied and $p_{i}>1, k \in \mathbb{N}$ are such that $q_{i}=$ $\frac{p_{i}}{k p_{i}-1}$ for $1 \leq i \leq n$. Let

$$
1+\frac{u_{i}(x) w^{\prime}(x)}{\left(1-\alpha k p_{i}\right) w(x) u_{i}^{\prime}(x)} \geq \frac{1}{\gamma_{i}}>0 \text { (a.e), for } \alpha>\frac{1}{k p_{i}} \text { and } 1 \leq i \leq n
$$




$$
1+\frac{u_{i}(x) w^{\prime}(x)}{\left(\alpha k p_{i}-1\right) w(x) u_{i}^{\prime}(x)} \geq \frac{1}{\delta_{i}}>0 \quad \text { (a.e), for } \alpha<\frac{1}{k p_{i}} \text { and } 1 \leq i \leq n .
$$

Then,

$$
\sum_{i=1}^{n-k+2} \int_{0}^{\infty} w(x)\left[\prod_{j=i}^{i+k-1} R_{j}^{p_{j}}(x)\right] d x \leq \sum_{i=1}^{n}\left[\frac{k p_{i} \lambda_{i}}{\left|\alpha k p_{i}-1\right|}\right]^{k p_{i}} \int_{0}^{\infty} w(x) g_{i}(x) d x
$$

where,

$$
\begin{gathered}
g_{i}(x) \mapsto \frac{u_{i}^{2-\alpha}(x) z_{i}^{\prime}(x) f_{i}(x)}{z_{i}(x) \sqrt[k p_{i}]{u_{i}^{\prime}(x)}} ; \quad \lambda_{i}=\max _{1 \leq i \leq n}\left(\gamma_{i}, \delta_{i}\right), \quad n \geq k-1 \text { and } \\
R_{i}(x)=\left\{\begin{array}{l}
\frac{k p_{i} \sqrt{u_{i}^{\prime}(x)}}{u_{i}^{\alpha}(x)} \int_{0}^{x} \frac{u_{i}(t) z_{i}^{\prime}(t)}{z_{i}(t)} f_{i}(t) d t, \quad \alpha>\frac{1}{k p_{i}} ; \\
\frac{k p_{i} \sqrt{u_{i}^{\prime}(x)}}{u_{i}^{\alpha}(x)} \int_{x}^{\infty} \frac{u_{i}(t) z_{i}^{\prime}(t)}{z_{i}(t)} f_{i}(t) d t, \quad \alpha<\frac{1}{k p_{i}} .
\end{array}\right.
\end{gathered}
$$

Proof. Let us define for $\alpha>\frac{1}{k p_{i}}, 1 \leq i \leq n 0 \leq x \leq X$ and $0<a<b<\infty$ :

$$
R_{i a}(x)=\frac{\sqrt[k p_{i}]{u_{i}^{\prime}(x)}}{u_{i}^{\alpha}(x)} \int_{a}^{x} \frac{u_{i}(t) z_{i}^{\prime}(t)}{z_{i}(t)} f_{i}(t) d t, \quad 1 \leq i \leq n .
$$

By using the inequality (3) for $C_{i}=R_{i a}^{p_{i}}(x)$

$$
\sum_{i=1}^{n-k+2} R_{i a}^{p_{i}}(x) R_{(i+1) a}^{p_{i+1}}(x) \ldots R_{(i+k-1) a}^{p_{i+k-1}}(x) \leq \sum_{i=1}^{n} R_{i a}^{k p_{i}}(x),
$$

i.e.,

$$
\sum_{i=1}^{n-k+2} \int_{a}^{b} w(x)\left[\prod_{j=i}^{i+k-1} R_{j a}^{p_{j}}(x)\right] d x \leq \sum_{i=1}^{n} \int_{a}^{b} w(x) R_{i a}^{k p_{i}}(x) d x .
$$

Now consider,

$$
\begin{aligned}
I & =\int_{a}^{b} w(x) R_{i a}^{k p_{i}}(x) d x \\
& =\int_{a}^{b} u_{i}^{-\alpha k p_{i}}(x) u_{i}^{\prime}(x)\left[\sqrt[k p_{i}]{w(x)} \int_{a}^{x} \frac{u_{i}(t) z_{i}^{\prime}(t)}{z_{i}(t)} f_{i}(t) d t\right]^{k p_{i}} d x .
\end{aligned}
$$

Integrating by parts, we have

$$
\begin{aligned}
I= & \left|\frac{u_{i}^{1-k p_{i} \alpha}(x)}{1-k p_{i} \alpha}\left[\sqrt[k p_{i}]{w(x)} \int_{a}^{x} \frac{u_{i}(t) z_{i}^{\prime}(t)}{z_{i}(t)} f_{i}(t) d t\right]_{a}^{k p_{i}}\right|_{a}^{b} \\
& -\int_{a}^{b} \frac{u_{i}^{1-k p_{i} \alpha}(x)}{1-k p_{i} \alpha} \frac{d}{d x}\left(\sqrt[k p_{i}]{w(x)} \int_{a}^{x} \frac{u_{i}(t) z_{i}^{\prime}(t)}{z_{i}(t)} f_{i}(t) d t\right)^{k p_{i}} d x
\end{aligned}
$$




$$
\begin{aligned}
= & \frac{w(b) u_{i}^{1-k p_{i} \alpha}(b)}{1-k p_{i} \alpha}\left[\int_{a}^{b} \frac{u_{i}(t) z_{i}^{\prime}(t)}{z_{i}(t)} f_{i}(t) d t\right]^{k p_{i}}-\frac{1}{1-k p_{i} \alpha} \int_{a}^{b} \frac{u_{i}(x) w^{\prime}(x) R_{i a}^{k p_{i}}(x)}{u_{i}^{\prime}(x)} d x \\
& -\frac{k p_{i}}{1-k p_{i} \alpha} \int_{a}^{b} \frac{u_{i}^{2-\alpha}(x) w(x) z_{i}^{\prime}(x) f_{i}(x) R_{i a}^{k p_{i}-1}(x)}{z_{i}(x) \sqrt[k p_{i}]{u_{i}^{\prime}(x)}} d x
\end{aligned}
$$

From (12) and (16) we have

$$
I=\int_{a}^{b} w(x) R_{i a}^{k p_{i}}(x) d x \leq \frac{\gamma_{i} k p_{i}}{k p_{i} \alpha-1} \int_{a}^{b} \frac{u_{i}^{2-\alpha}(x) w(x) z_{i}^{\prime}(x) f_{i}(x) R_{i a}^{k p_{i}-1}(x)}{z_{i}(x) \sqrt[k p_{i}]{u_{i}^{\prime}(x)}} d x .
$$

By Hölder's inequality:

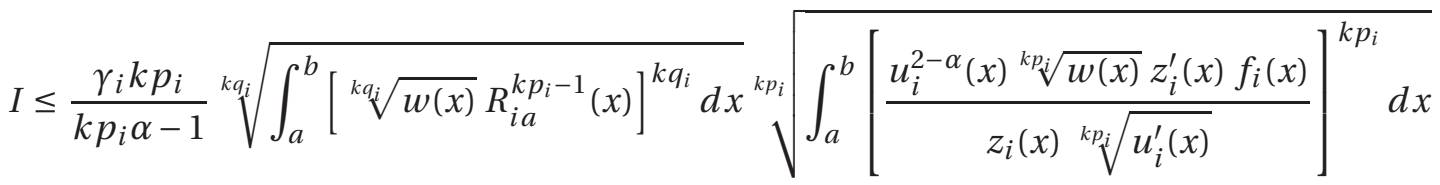

$$
\begin{aligned}
& \leq\left[\frac{\gamma_{i} k p_{i}}{k p_{i} \alpha-1}\right]^{k p_{i}} \int_{a}^{b} \frac{w(x)}{u_{i}^{\prime}(x)}\left[\frac{u_{i}^{2-\alpha}(x) z_{i}^{\prime}(x) f_{i}(x)}{z_{i}(x)}\right]^{k p_{i}} d x .
\end{aligned}
$$

From (15) and (18) we have

$$
\begin{aligned}
\sum_{i=1}^{n-k+2} \int_{a}^{b} w(x)\left[\prod_{j=i}^{i+k-1} R_{j a}^{p_{j}}(x)\right] d x & \leq \sum_{i=1}^{n}\left[\frac{k p_{i} \lambda_{i}}{\alpha k p_{i}-1}\right]^{k p_{i}} \int_{a}^{b} w(x) g_{i}(x) d x \\
& \leq \sum_{i=1}^{n}\left[\frac{k p_{i} \lambda_{i}}{\alpha k p_{i}-1}\right]^{k p_{i}} \int_{a}^{\infty} w(x) g_{i}(x) d x
\end{aligned}
$$

Let us define for $\alpha<\frac{1}{k p_{i}}$ and $0<a<b<\infty$ :

$$
R_{i b}(x)=\frac{\sqrt[k p_{i}]{u_{i}^{\prime}(x)}}{u_{i}^{\alpha}(x)} \int_{x}^{b} \frac{u_{i}(t) z_{i}^{\prime}(t)}{z_{i}(t)} f_{i}(t) d t, \quad 1 \leq i \leq n .
$$

Following the same steps as in the proof of inequality (19) we obtain

$$
\begin{aligned}
\sum_{i=1}^{n-k+2} \int_{a}^{b} w(x)\left[\prod_{j=i}^{i+k-1} R_{j a}^{p_{j}}(x)\right] d x & \leq \sum_{i=1}^{n}\left[\frac{k p_{i} \lambda_{i}}{1-\alpha k p_{i}}\right]^{k p_{i}} \int_{a}^{b} w(x) g_{i}(x) d x \\
& \leq \sum_{i=1}^{n}\left[\frac{k p_{i} \lambda_{i}}{1-\alpha k p_{i}}\right]^{k p_{i}} \int_{a}^{\infty} w(x) g_{i}(x) d x
\end{aligned}
$$

By letting $a \rightarrow 0$ and $b \rightarrow \infty$ in inequalities (19) and (20) we get (14).

Corollary 2. For any $1 \leq i \leq n$, let $w, h_{i}:[0, X] \rightarrow \mathbb{R}_{+}$absolutely continuous functions for $X \in \mathbb{R}_{+}$ and $\lambda_{i}=\max _{1 \leq i \leq n}\left(\gamma_{i}, \delta_{i}\right)$. Let

$$
1+\frac{x w^{\prime}(x)}{\left(1-2 \alpha p_{i}\right) w(x)} \geq \frac{1}{\gamma_{i}}>0, \text { for } \alpha>\frac{1}{2 p_{i}} \text { and } 1 \leq i \leq n .
$$




$$
1+\frac{x w^{\prime}(x)}{\left(2 \alpha p_{i}-1\right) w(x)} \geq \frac{1}{\delta_{i}}>0, \text { for } \alpha<\frac{1}{2 p_{i}} \text { and } 1 \leq i \leq n
$$

Then,

$$
\sum_{i=1}^{n} \int_{0}^{\infty} w(x)\left[\frac{1}{x^{\alpha}} \int_{a}^{x} t^{\alpha-1} h_{i}(t) d t\right]^{p_{i}} d x \leq \sum_{i=1}^{n}\left[\frac{2 p_{i} \lambda_{i}}{2 \alpha p_{i}-1}\right]^{2 p_{i}} \int_{0}^{\infty} w(x) h_{i}(x) d x
$$

Proof. Follows from Theorem 2 by setting $z_{i}(t)=u_{i}(t)=t ; h_{i}(t)=h_{i+1}(t) ; p_{i}=p_{i+1}$ and $f_{i}(t) \mapsto t^{\alpha-1} h_{i}(t)$ for $k=2$ and $1 \leq i \leq n$.

Theorem 3. Let the conditions of Theorem 1 be satisfied and $p_{i}>1$, is such that $q_{i}=\frac{p_{i}}{3 p_{i}-1}$ for $1 \leq i \leq n$. Let

$$
1+\frac{u_{i}(x) w^{\prime}(x)}{\left(1-3 \alpha p_{i}\right) w(x) u_{i}^{\prime}(x)} \geq \frac{1}{\gamma_{i}}>0 \text { (a.e), for } \alpha>\frac{1}{3 p_{i}} \text { and } 1 \leq i \leq n .
$$

Then,

$$
\sum_{i=1}^{n-1} \int_{0}^{X} w(x) F_{i}^{p_{i}}(x) F_{i+1}^{p_{i+1}}(x) F_{i+2}^{p_{i+2}}(x) d x \leq \sum_{i=1}^{n}\left[\frac{3 p_{i} \gamma_{i}}{3 \alpha p_{i}-1}\right]^{3 p_{i}} \int_{0}^{X} w(x) g_{i}(x) d x,
$$

where,

$$
\begin{aligned}
& g_{i}(x) \mapsto\left[\frac{u_{i}^{2-\alpha}(x) z_{i}^{\prime}(x) f_{i}(x)}{z_{i}(x) \sqrt[3 q_{i}]{u_{i}^{\prime}(x)}}-\frac{u_{i}^{1-\alpha}(x) u_{i}\left(\frac{x}{2}\right) z_{i}^{\prime}\left(\frac{x}{2}\right) f_{i}\left(\frac{x}{2}\right)}{2 z_{i}\left(\frac{x}{2}\right) \sqrt[3 q_{i}]{u_{i}^{\prime}(x)}}\right]^{3 p_{i}} \text { and } \\
& F_{i}(x)=\frac{\sqrt[3 p_{i}]{u_{i}^{\prime}(x)}}{u_{i}^{\alpha}(x)} \int_{x / 2}^{x} \frac{u_{i}(t) z_{i}^{\prime}(t)}{z_{i}(t)} f_{i}(t) d t .
\end{aligned}
$$

Proof. Let us define for $\alpha>\frac{1}{3 p_{i}}, 1 \leq i \leq n, 0 \leq x \leq X$ :

$$
F_{i}(x)=\frac{\sqrt[3 p_{i}]{u_{i}^{\prime}(x)}}{u_{i}^{\alpha}(x)} \int_{x / 2}^{x} \frac{u_{i}(t) z_{i}^{\prime}(t)}{z_{i}(t)} f_{i}(t) d t, 1 \leq i \leq n .
$$

By using the inequality (3) with $k=3$ for $C_{i}=F_{i}^{p_{i}}(x)$ :

$$
\sum_{i=1}^{n-1} F_{i}^{p_{i}}(x) F_{i+1}^{p_{i+1}}(x) F_{i+2}^{p_{i+2}}(x) \leq \sum_{i=1}^{n} F_{i}^{3 p_{i}}(x)
$$

i.e.,

$$
\sum_{i=1}^{n-1} \int_{0}^{X} w(x) F_{i}^{p_{i}}(x) F_{i+1}^{p_{i+1}}(x) F_{i+2}^{p_{i+2}}(x) d x \leq \sum_{i=1}^{n} \int_{0}^{X} w(x) F_{i}^{3 p_{i}}(x) d x .
$$

Now consider

$$
I=\int_{0}^{X} w(x) F_{i}^{3 p_{i}}(x) d x
$$




$$
=\int_{0}^{X} u_{i}^{-3 \alpha p_{i}}(x) u_{i}^{\prime}(x)\left[\sqrt[3 p_{i}]{w(x)} \int_{x / 2}^{x} \frac{u_{i}(t) z_{i}^{\prime}(t)}{z_{i}(t)} f_{i}(t) d t\right]^{3 p_{i}} d x
$$

Integrating by parts, we have

$$
\begin{aligned}
I= & \left|\frac{u_{i}^{1-3 p_{i} \alpha}(x)}{1-3 p_{i} \alpha}\left[\sqrt[3 p_{i}]{w(x)} \int_{x / 2}^{x} \frac{u_{i}(t) z_{i}^{\prime}(t)}{z_{i}(t)} f_{i}(t) d t\right]_{0}^{3 p_{i}}\right|_{0}^{X} \\
& -\int_{0}^{X} \frac{u_{i}^{1-3 p_{i} \alpha}(x)}{1-3 p_{i} \alpha} \frac{d}{d x}\left(\sqrt[3 p_{i}]{w(x)} \int_{x / 2}^{x} \frac{u_{i}(t) z_{i}^{\prime}(t)}{z_{i}(t)} f_{i}(t) d t\right)^{3 p_{i}} d x \\
= & \frac{w(X) u_{i}(X) F_{i}^{3 p_{i}}(X)}{u_{i}^{\prime}(X)\left(1-3 p_{i} \alpha\right)}-\int_{0}^{X} \frac{w^{\prime}(x) u_{i}(x) F_{i}^{3 p_{i}}(x)}{u_{i}^{\prime}(x)\left(1-3 p_{i} \alpha\right)} d x-\frac{3 p_{i}}{1-3 p_{i} \alpha} \int_{0}^{X} w(x) \\
& \times F_{i}^{3 p_{i}-1}(x)\left[\frac{u_{i}^{2-\alpha}(x) z_{i}^{\prime}(x) f_{i}(x)}{z_{i}(x) \sqrt[3 q_{i}]{u_{i}^{\prime}(x)}}-\frac{u_{i}^{1-\alpha}(x) z_{i}^{\prime}(x / 2) f_{i}(x / 2) u_{i}(x / 2)}{2 z_{i}(x / 2) \sqrt[3 q_{i}]{u_{i}^{\prime}(x)}}\right] d x .
\end{aligned}
$$

From (21) and (24) we have

$$
\begin{aligned}
& \int_{0}^{X} w(x) F_{i}^{3 p_{i}}(x) d x \\
& \leq \frac{3 p_{i} \gamma_{i}}{1-3 p_{i} \alpha} \int_{0}^{X} w(x) F_{i}^{3 p_{i}-1}(x)\left[\frac{u_{i}^{2-\alpha}(x) z_{i}^{\prime}(x) f_{i}(x)}{z_{i}(x) \sqrt[3 q_{i}]{u_{i}^{\prime}(x)}}-\frac{u_{i}^{1-\alpha}(x) z_{i}^{\prime}\left(\frac{x}{2}\right) f_{i}\left(\frac{x}{2}\right) u_{i}\left(\frac{x}{2}\right)}{2 z_{i}\left(\frac{x}{2}\right) \sqrt[3 q_{i}]{u_{i}^{\prime}(x)}}\right] d x .
\end{aligned}
$$

By Hölder's inequality:

$$
\begin{aligned}
& I \leq \frac{3 \gamma_{i} p_{i}}{3 p_{i} \alpha-1} \sqrt[3 q_{i}]{\int_{0}^{X}\left[\sqrt[3 q_{i}]{w(x)} F_{i}^{3 p_{i}-1}(x)\right]^{3 q_{i}} d x} \\
& \times \sqrt[3 p_{i}]{\int_{0}^{X} w(x)\left[\frac{u_{i}^{2-\alpha}(x) z_{i}^{\prime}(x) f_{i}(x)}{z_{i}(x) \sqrt[3 q_{i}]{u_{i}^{\prime}(x)}}-\frac{u_{i}^{1-\alpha}(x) z_{i}^{\prime}(x / 2) f_{i}(x / 2) u_{i}(x / 2)}{2 z_{i}(x / 2) \sqrt[3 q_{i}]{u_{i}^{\prime}(x)}}\right]^{3 p_{i}}} d x \\
& \leq\left[\frac{3 \gamma_{i} p_{i}}{3 p_{i} \alpha-1}\right]^{3 p_{i}} \int_{0}^{X} w(x)\left[\frac{u_{i}^{2-\alpha}(x) z_{i}^{\prime}(x) f_{i}(x)}{z_{i}(x) \sqrt[3 q_{i}]{u_{i}^{\prime}(x)}}-\frac{z_{i}^{\prime}(x / 2) f_{i}(x / 2) u_{i}(x / 2)}{2 u_{i}^{\alpha-1}(x) z_{i}(x / 2) \sqrt[3 q_{i}]{u_{i}^{\prime}(x)}}\right]^{3 p_{i}} d x .
\end{aligned}
$$

From (23) and (26) we get (22).

Lemma 2 ([9]). If $C_{1}, C_{2}, \ldots, C_{n}$ are reals and $C_{n+1}=C_{1}$ for $k \geq 1$, then

$$
\left[\sum_{r=1}^{n} C_{r}\right]^{k} \leq n^{k-1} \sum_{r=1}^{n} C_{r}^{k}
$$


Theorem 4. Let the conditions of Theorem 1 be satisfied and $p>1, k \geq 1$ are such that $q=\frac{p}{k p-1}$. If $\alpha>0$ and

$$
1+\frac{u_{i}(x) w^{\prime}(x)}{(1+k \alpha p) w(x) u_{i}^{\prime}(x)} \geq \frac{1}{\gamma_{i}}>0(\text { a.e }), \text { for } 1 \leq i \leq n .
$$

Then,

$$
\int_{a}^{b} w(x)\left[\sum_{i=1}^{n} F_{i a}(x)\right]^{k p} d x \leq \sum_{i=1}^{n}\left[\frac{k p \gamma_{i} \sqrt[k q]{n}}{k \alpha p+1}\right]^{k p} \int_{a}^{b} w(x) g_{i}(x) d x
$$

where,

$$
g_{i}(x) \mapsto \frac{u_{i}^{k p \alpha}(x)\left[z_{i}^{\prime}(x)\right]^{k p} f_{i}^{k p}(x)}{\left[z_{i}(x)\right]^{k p}\left(u_{i}^{\prime}(x)\right)^{p / q}} \text { and } F_{i}(x)=u_{i}^{\alpha}(x) \sqrt[k p]{u_{i}^{\prime}(x)} \int_{0}^{x} \frac{z_{i}^{\prime}(t) f_{i}(t)}{u_{i}(t) z_{i}(t)} d t .
$$

Proof. Let us define for $1 \leq i \leq n ; 0 \leq x \leq X$ and $0<a<b<\infty$ :

$$
F_{i a}(x)=u_{i}^{\alpha}(x) \sqrt[k p]{u_{i}^{\prime}(x)} \int_{a}^{x} \frac{z_{i}^{\prime}(t) f_{i}(t)}{u_{i}(t) z_{i}(t)} d t, 1 \leq i \leq n .
$$

By using the inequality (27) for $C_{i}=F_{i a}(x)$ and $k \mapsto k p$ :

$$
\left[\sum_{i=1}^{n} F_{i a}(x)\right]^{k p} \leq n^{p k-1} \sum_{i=1}^{n} F_{i a}^{k p}(x)
$$

i.e.,

$$
\int_{a}^{b} w(x)\left[\sum_{i=1}^{n} F_{i a}(x)\right]^{k p} d x \leq n^{p k-1} \sum_{i=1}^{n} \int_{a}^{b} u_{i}^{k p \alpha}(x) u_{i}^{\prime}(x)\left[\sqrt[k p]{w(x)} \int_{a}^{x} \frac{z_{i}^{\prime}(t) f_{i}(t)}{u_{i}(t) z_{i}(t)} d t\right]^{k p} d x
$$

Now consider,

$$
I=\int_{a}^{b} u_{i}^{k p \alpha}(x) u_{i}^{\prime}(x) \times\left[\sqrt[k p]{w(x)} \int_{a}^{x} \frac{z_{i}^{\prime}(t) f_{i}(t)}{u_{i}(t) z_{i}(t)} d t\right]^{k p} d x .
$$

Integrating by parts, we have

$$
\begin{aligned}
I= & \left|\frac{u_{i}^{1+k p \alpha}(x)}{1+k p \alpha} w(x)\left[\int_{a}^{x} \frac{z_{i}^{\prime}(t) f_{i}(t)}{u_{i}(t) z_{i}(t)} d t\right]_{a}^{k p}\right|^{b} \\
& -\int_{a}^{b} \frac{u_{i}^{1+k p \alpha}(x)}{1+k p \alpha} \frac{d}{d x}\left(\sqrt[k p]{w(x)} \int_{a}^{x} \frac{f_{i}(t) z_{i}^{\prime}(t)}{u_{i}(t) z_{i}(t)} d t\right)^{k p} d x \\
= & \frac{w(b) u_{i}(b) F_{i a}^{k p}(b)}{u_{i}^{\prime}(b)(1+k p \alpha)}-\int_{a}^{b} \frac{w^{\prime}(x) u_{i}(x) F_{i a}^{k p}(x)}{u_{i}^{\prime}(x)(1+k p \alpha)} d x \\
& +\frac{k p}{1+k p \alpha} \int_{a}^{b} w(x) \frac{F_{i a}^{k p-1} u_{i}^{\alpha}(x) z_{i}^{\prime}(x) f_{i}(x)}{z_{i}(x) \sqrt[k g]{u_{i}^{\prime}(x)}} d x
\end{aligned}
$$


From (28) and (31) we have:

$$
\int_{a}^{b} w(x) F_{i a}^{k p}(x) d x \leq \frac{k p \gamma_{i}}{1+k p \alpha} \int_{a}^{b} w(x) \times \frac{F_{i a}^{k p-1} u_{i}^{\alpha}(x) z_{i}^{\prime}(x) f_{i}(x)}{z_{i}(x) \sqrt[k q]{u_{i}^{\prime}(x)}} d x
$$

By Hölder's inequality:

$$
\begin{aligned}
& I \leq \frac{k \gamma_{i} p}{k p \alpha+1} \sqrt[k q]{\int_{a}^{b}\left[\sqrt[k q]{w(x)} F_{i a}^{k p-1}(x)\right]^{k q} d x} \sqrt[k p]{\int_{a}^{b} w(x)\left[\frac{u_{i}^{\alpha}(x) z_{i}^{\prime}(x) f_{i}(x)}{z_{i}(x) \sqrt[k q]{u_{i}^{\prime}(x)}}\right]^{k p}} d x \\
& \leq\left[\frac{k \gamma_{i} p}{k p \alpha+1}\right]^{k p} \int_{a}^{b} \frac{w(x) u_{i}^{k p \alpha}(x)\left[z_{i}^{\prime}(x)\right]^{k p} f_{i}^{k p}(x)}{z_{i}^{k p}(x)\left[u_{i}^{\prime}(x)\right]^{p / q}} d x .
\end{aligned}
$$

From (30) and (33) we get (29).

Corollary 3. For any $1 \leq i \leq n$, let $w, h_{i}:[a, b] \rightarrow \mathbb{R}_{+}$be absolutely continuous functions for $0<a<b<\infty$ and $p>1$. If

$$
1+\frac{x w^{\prime}(x)}{(1+k \alpha p) w(x)} \geq \frac{1}{\gamma_{i}}>0, \text { for } \alpha>\frac{1}{2 p} \text { and } 1 \leq i \leq n .
$$

Then,

$$
\int_{a}^{b} w(x)\left[\sum_{i=1}^{n} x^{\alpha} \int_{a}^{x} \frac{h_{i}(t)}{t^{2}} d t\right]^{k p} d x \leq \sum_{i=1}^{n}\left[\frac{\sqrt[k q]{n} k p \gamma_{i}}{k \alpha p+1}\right]^{2 p_{i}} \int_{a}^{b} w(x) h_{i}^{k p}(x) d x .
$$

Proof. Follows from Theorem 4 by setting $z_{i}(t)=u_{i}(t)=t$ and $f_{i}(t) \mapsto t^{1-\alpha} h_{i}(t)$ for $1 \leq i \leq n$.

\section{References}

[1] W. S. Cheung, Z. Hanjs and J. Pečarić, Some Hardy-type inequalities, J. Math. Anal. Appl., 250(2000), 621-634.

[2] S. Hussain, M. A. Latif and M. Iqbal, Multidimensional Weighted Boas type inequality and related results with applications, Rocky Mountain Journal of Mathematics (to appear).

[3] S. Hussain and J. Pečarić, Bounds for Multidimensional Hardy type Polya-Knopp differences, Inequality Theory and Applications, Vol. 6, Nova Science Pub Inc, 2010, 213-231. ISBN: 978-1616686253.

[4] S. Hussain and J. Pečarić, Bounds for Strengthened Hardy and Polya-Knopp's difference, Rocky Mountain J. Math., 40 (2010), 913-928.

[5] S. Hussain and J. Pečarić, Bounds for Hardy differences, Anziam J., 52(2010), 218-224. doi:10.1017/S1446181111000642.

[6] G. H. Hardy, J. E. Littlewood and G. Polya, Inequalities, Cambridge Univ. Press, Cambridge, 1952.

[7] G. H. Hardy, Notes on some points in the integral calculus, Messenger Math., 57 (1928), 12-16.

[8] G. H. Hardy, Notes on a theorem of Hilbert, Math. Z., 6 (1920), 314-317.

[9] D. S. Mitrinović, Analytic Inequalities Berlin: Springer, 1970.

[10] B. G. Pachpatte, On some variants of Hardy's inequality, J. Math. Anal. Appl., 124 (1987), 495-501.

[11] B. G. Pachpatte, On some generalizations of Hardy's integral inequality, J. Math. Anal. Appl., 234(1999), 15-30. 
[12] B. G. Pachpatte, On a new class of Hardy type inequalities, Proc. R. Soc. Edin., 105A (1987), 265-274.

[13] J. E. Pečarić and E. R. Love, Still more generalizations of Hardy's inequality, J. Austral. Math. Soc. Ser. A, 58 (1995), 1-11.

[14] B. Yang, Z. Zeng and L. Debnath, On New Generalizations of Hardy's Integral Inequality, J. Math. Anal. Appl., 217(1998), 321-327.

Department of Mathematics, University of Engineering and Technology, Lahore, Pakistan.

E-mail: sabirhus@gmail.com

Department of Mathematics, University of Hail, Hail, Saudi Arabia.

E-mail: m_amer_latif@hotmail.com

Institute of Space Technology, Near Rawat Tool Plaza, Islamabad Highway, Islamabad.

E-mail: Wasakh75@hotmail.com 